\title{
Blur Detection Methods for Digital Images-A Survey
}

\author{
Rupali Yashwant Landge \\ Department of Computer Science and Engineering \\ Rajasthan college of Engineering for women, Jaipur
}

\author{
Rakesh Sharma \\ Department of Computer Science and Engineering \\ Rajasthan college of Engineering for women, Jaipur
}

\begin{abstract}
This paper described various blur detection methods along with proposed method. Digital photos are massively produced while digital cameras are becoming popular; however, not every photo has good quality. Blur is one of the conventional image quality degradation which is caused by various factors like limited contrast; inappropriate exposure time and improper device handling indeed, blurry images make up a significant percentage of anyone's picture collections. Consequently, an efficient tool to detect blurry images and label or separate them for automatic deletion in order to preserve storage capacity and the quality of image collections is needed. There are various methods to detect the blur from the blurry images some of which requires transforms like DCT or Wavelet and some doesn't require transform.
\end{abstract}

Keywords: Blur, DCT, DWT, SIFT, Wavelet.

\section{INTRODUCTION}

Advances in computational photography over the last decade have laid the foundations for the mass production of powerful low-cost digital cameras. This technology is indeed helping conventional users to generate high-quality content with inexpensive and bulky professional cameras. The Latest generation offers cameras with, autofocusing and motion compensation functions. The aim of such functions is to improve picture quality by automatically post-processing and enhancing the quality of images captured with low-quality, low-price sensors and lenses. High-quality lenses and sensors are not only expensive but bulky and thus inappropriate for integration in small cameras and other devices such as mobile handsets. Computational photography offers highly efficient tools that can greatly improve the quality of pictures captured with low-quality lenses and sensors at very low cost.This approach offers a very appealing alternative to image capturing with high-quality lenses. Unfortunately, not all the shortcomings of low-cost digital cameras can be yet compensated by software post-processing. Indeed, simple factors such as limited light conditions, inappropriate exposure time and improper device handling can lead to unsatisfactory image quality. As a consequence, the search for better image enhancement and selection tools in the field of computational photography goes on.

Nowadays, high-quality digital cameras gain increasing attention as digital technology advances. Users can take hundreds of pictures a day. However, it is not easy for them to look through all their pictures to decide which of them can be deleted (for example, if the storage is full), or which of them should be taken for an enhancement process. Thus, they need some techniques of image quality estimation for telling the blurry images from the sharp ones. Imperfect focusing and/or motion is the main source of blurriness in digital photographs. Clearly, blurry images make up a significant percentage of anyone's picture collections captured with conventional digital cameras in usual conditions. As a consequence, a tool to automatically detect blurry images is urgently needed. The reason is twofold. On the one hand, blurry images can be labeled automatically and separated from good-quality images in conventional collections for browsing, viewing and later re-use. On the other hand, the same functionality can be used for automatic deletion in order to preserve storage capacity in the flash memory of a digital camera. The latter feature will enable users to virtually increase the storage capacity of their cameras by retaining only those pictures with perceptively good quality [1]. There are already some existing methods for blur detection or image quality estimation for digital images. However, most of them are time-consuming, computation intensive, need different kinds of transformations (e.g. DCT or DWT) or the detection ratio is not very high .Also there is one proposed algorithm for automatic real time detection of blurry images The algorithm is based on computing variance values of the local key points that are extracted from the given images through implementing Scale Invariant Feature Transform (SIFT) algorithm in a scale space. No transforms (DCT or DWT) are required to be applied to the images, and no edge locations need to be identified in the proposed method, which are the main techniques used in most of the existing methods. Only pixel values of the given images are directly employed in the algorithm [2]. The main objective is to automatically detect blurry images for automatic labeling and potential removal. The targeted application is low-cost digital cameras for conventional users. The presented approach is generic in the sense that it does not make assumptions on the cause of blurriness.

\section{EXISTING BLUR DETECTION TECHNIQUES}

Following are the existing blur detection techniques. 


\subsection{Blur Detection for Digital Images Using Wavelet Transform:}

Blur detection scheme using Harr wavelet transform is a direct methods. It can not only judge whether or not a given image is blurred, which is based on edge type analysis, but also determine to what extent the given image is blurred, which is based on edge sharpness analysis[3]. The scheme takes advantage of the ability of Harr wavelet transform in both discriminating different types of edges and recovering sharpness from the blurred version. It is effective for both Out-of-focus blur and Linear-motion blur. Its effectiveness will not be affected by the uniform background in images.

Different edges are generally classified into three types: namely, Dirac-Structure, Step-Structure and Roof-Structure .Step-Structure is further classify into Astep-Structure and Gstep- Structure according to whether the change of intensity is gradual or not. for Gstep- Structure and Roof-Structure edge, there is a parameter $a(0<a<n / 2)$ indicating the sharpness of the edge: the larger $a$ is, the sharper the edge is. The basic idea of the scheme is : In general, most natural images contain all types of edges more or less, and most Gstep-Structure and Roof- Structure are sharp enough. When blur occurs, no matter whether it is caused by Out-of-focus or Linear motion, both Dirac-Structure and Astep-Structure will disappear. What is more, both Gstep Structure and RoofStructure tend to lose their sharpness.

The scheme judges whether a given image is blurred according to whether it has Dirac-Structure or AstepStructure, and uses the percentage of Gstep-Structure and Roof-Structure which are more likely to be in a blurred image to determine the blur extent. The whole structure of the scheme is shown in Fig. 1.

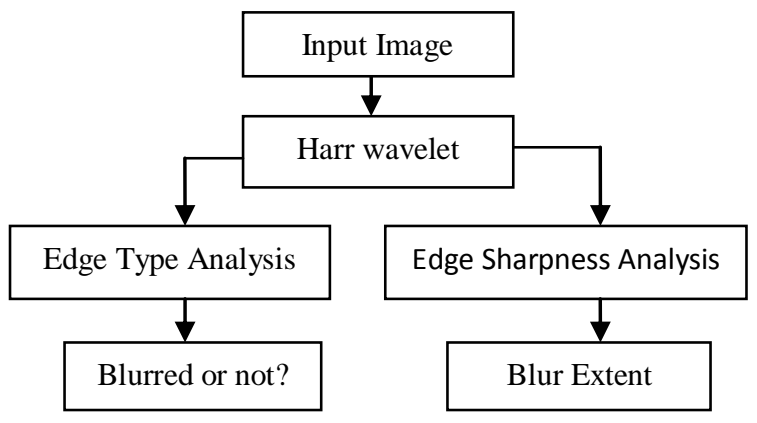

Fig.1 Structure of the HWT blur detection scheme

\subsection{Blur Detection for Digital Images Using DCT:}

Blur detection for DCT uses a new solution to aim at exploiting the available DCT information in MPEG or JPEG compressed video or images while involving a minimal computational load, the technique is based on histograms of non-zero DCT occurrences, computed directly from MPEG or JPEG compressed images. For MPEG compressed video, the scheme is suitable for all types of pictures: I-frames, P-frames or B-frames.

The objective of blur detection in this application is to provide a percentage indicating the global image quality in terms of blur: $0 \%$ would mean that the frame is totally blurred while $100 \%$ would mean that no blur at all is present in that particular frame. This blur indicator characterizes the global image blur caused by camera motion or out of focus. Since we focus analyzing MPEG compressed video data, it is desirable that the blur indicator can be directly derived from the DCT layer of an MPEG video bit stream. To achieve this objective, one should be aware that:

a) The DCT coefficients used within MPEG are intended for compression and are deeply related to the image content. Basically, they reflect the frequency distribution of an image block.

b) In a MPEG stream, DCT coefficients are directly applied on the pixels of I-frames. On the contrary, coefficients of Pand $\mathrm{B}$-frames describe the residual image that remains after motion compensation [4].

It is therefore important to select a blur indicator which is as independent as possible from the particular content of an image as well as from the type of MPEG frames (I, P or B).

Blur is the opposite of edge sharpness. DCT coefficients render this sharpness via the high values of some AC coefficients. In this method blur measure therefore looks for the absence of such edges into the image, which is considered to prove a blurred image. Following three steps lead to the final measure:

Step 1. In order to characterize the global blur, it is proposed to establish a measure that takes into account the DCT information of the entire image as a whole. It is likely that any type of edge will cross some $8 \times 8$ blocks at least once in the image. Globalization among all DCT blocks would therefore enable to have an idea about the general edge sharpness, i.e. the global (camera or motion) blur.

Step 2. In order to be as independent as possible of the content of the image, coefficients should not be considered directly since their values are closely related to the type of image they depict. One rather proposes to look at the distribution of null coefficients instead of the values themselves: blurred images are likely to have all of their high frequency coefficients set to zero, whatever their content is.

Step 3. In order to remove the dependency to the image size, the number of blocks in the image should divide the number of times a coefficient is not zero. This would limit histogram values to 1 . However, coefficients are often zeros in P- and Bframes. In order to homogenize the look of the histogram for all types of pictures, the number of non-zero occurrences of a coefficient is divided by the number of non-zero occurrences of the DC coefficient. 
With these three steps, a measure (histogram) is obtained which is independent of both the image content and the type of MPEG frames.

\subsection{Simple method for blur detection without using transform:}

As a pre-processing, only converting the input images from RGB colours to grey-level luminance values is needed for the tool described below.

Following are the steps for above method

1) Input image array as IMn

2) Check if array is two dimensional or not, if not convert it

3) Calculate global invariance value $S 2 p$ for different sample values

4) S2 p1 is sample variance value of the pre-image and $\mathrm{S} 2 \mathrm{p} 2$ is sample variance value of the taken image,

5) if image is first one only i.e. if $n=1$ and $S 2 p 1=S 2 p$, Go for the next image

6) If not then calculate ratio $R$ of sample variance values of pre-image and taken image.

7) If $R=1$ or $R<1$ then image is blur, if want to delete then delete it

8) If not then image is not blur.

\section{PROPOSED METHOD}

In order to evaluate images, first calculate variance values of the local key points in consecutive images and then to evaluate the differences of these variances. For this reason SIFT operator had been chosen to detect the objects and find their special features. Moreover, after obtaining the locations of image local key points, select only fixed amount of samples for further calculations. This helps to speed up the process of image evaluation.

In order to estimate images, first apply only one part of the SIFT algorithm, that is, detecting local key points of the images objects. Then, generate additional images from the given one through the linear diffusion process. And finally, analyse the variance values calculated for the local key points of the original and its filtered images generated in the scale space. The variance value is calculate using formula given below:

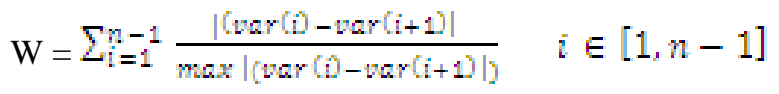

The algorithm for proposed method is described as follows:

1) input image;
2) apply SIFT to obtain key points of the image;

3) randomly select a certain amount of the key points from the given image and fixed location;

4) apply scale-space low-pass Gaussian filtering to the original image;

5) take the key points in the filtered images from the same locations fixed previously in the original image;

6) calculate variances for the taken key points in the original and filtered images and build the plot of it;

7) Analyze the curvature given in the plot.

The SIFT operator provides the number of key points found in the image and their position information. The number of key points varies from several hundreds to even hundreds of thousands per one image depending on the quality and structure of the image.

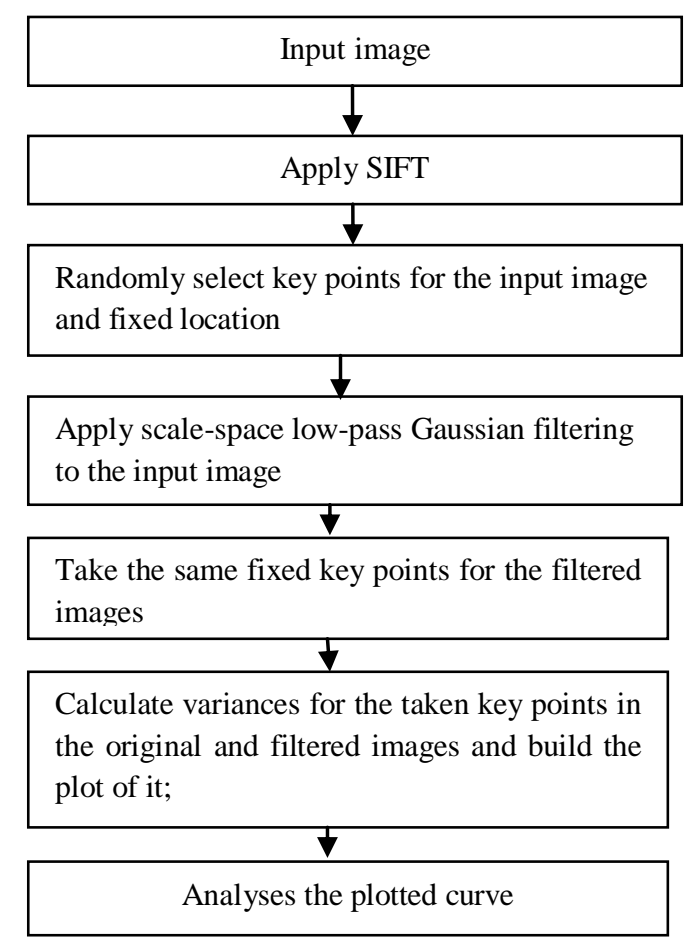

Fig 2.flowchart of proposed algorithm

\section{CONCLUSION}

The Harr wavelet scheme judges whether or not an image is blurred according to whether or not it contains any Dirac-Structure or Astep-Structure. Blur extent is characterized by blur confident coefficient based on the percentage of Gstep-Structure and Roof-Structure which are more likely to be in a blurred image. The scheme is effective and efficient. The DCT technique is directly applicable in the JPEG or MPEG compressed image without decompression. The method is effective for any type of images (I-, P- or B- 
frames). The blur detection approach without using transform is simple, highly efficient and very accurate and fast than the other two i.e. using DCT and Harr wavelet transform. It is suitable for fully automated and real-time applications. The algorithm proposed in this paper is applicable for real digital cameras. No additional 'pre-images' are required in the method. Only taken pictures should be processed in the camera.

\section{REFERENCES}

[1] E. Tsomko H.J. Kim, E. Izquierdo "Linear Gaussian blur evolution for detection of blurry images" IET image process.,2010 Vol. 4,ISS.4pp.302-312

[2] Prasad D.Pulekar "Blur Detection in Digital Images-A Survey.

[3] Tong H., Mingjing L., Hongjiang Z., Changshui Z.:'Blur detection for digital images using wavelet transform'. IEEE Int. Conf. on Multimedia and Expo (ICME), 2004, pp. 17-20.

[4] X. Marichal, W. Y Ma, and H. J. Zhang, "Blur determination in the compressed domain using DCT information,"Proceedings of the IEEE International Conference on Image Processing, pp 386-390, 1999.

[5] LOWE D.G.: 'Distinctive image features from scale invariant keypoints', Int. J. Comput. Vis., 2004, 60, pp. 91-110

[6] VEDALDI A. 'An open implementation of SIFT detector and descriptor'. UCLA CSD Technical Report, 070012, 2006

[7] ZHANG Q., CHEN Y., ZHANG Y., XU Y.: 'SIFT implementation and optimization for multi-core systems'. IEEE Int. Symp. on Parallel and Distributed Processing, 2008, pp. 1-8

[8] LIU R., LI Z., JIA J.: 'Image partial blur detection and classification'. Proc. IEEE Conf. Computer Vision and Pattern Recognition, 2008, pp. 1-8.

[9] BOULT B.E., CHIANG M.C.: 'Local blur estimation and super- resolution'. Proc. IEEE Conf. Computer Vision and Pattern Recognition, 1997, pp. 821-826

[10] TICO M., TRIMECHE M., VEHVILAINEN M.: 'Motion blur identification based on differently exposed images'. IEEE Int. Conf. Image Processing, 2006, pp. 2021-2024
[11] TSOMKO E., KIM H.J.: 'Efficient method of detecting globally blurry or sharp images'. Proc. Ninth Int. Workshop on Image Analysis for Multimedia Interactive Services (WIAMIS), Klagenfurt, Austria, May 2008, pp. 171-174

[12] MARZILIANO P., DUFAUX F., WINKLER S., EBRAHIMI T.: 'Perceptual blur and ringing metrics: application to JPEG 2000', Signal Process.: Image Commun., 2004, 19, pp. $163-172$ 